\title{
FATORES COMPORTAMENTAIS ASSOCIADOS À ADESÃO MEDICAMENTOSA EM IDOSOS EM ATENDIMENTO AMBULATORIAL
}

\section{BEHAVIORAL FACTORS ASSOCIATED TO MEDICATION ADHESION IN ELDERLY IN OUTPATIENT CARE}

\section{FACTORES CONDUCTUALES ASOCIADOS A LA ADHESIÓN DE FÁRMACOS EN LOS ANCIANOS EN ATENCIÓN AMBULATORIA}

\author{
Daiane Porto Gauterio Abreu ${ }^{1}$, Silvana Sidney Costa Santos ${ }^{2}$, Silomar Ilha ${ }^{3}$, Bárbara Tarouco da Silva ${ }^{4}$, Nidia Farias Fernandes \\ Martins ${ }^{5}$, Victorya dos Santos Varela ${ }^{6}$.
}

\section{RESUMO}

Objetivo: avaliar a relação entre fatores comportamentais e adesão à terapêutica medicamentosa em idosos em atendimento ambulatorial. Método: estudo transversal com amostra de 107 idosos em atendimento ambulatorial em um hospital universitário. Os dados foram coletados por meio de entrevista. Aplicaram-se: instrumento de caracterização do idoso, Miniexame do Estado Mental e Medida de Adesão aos Tratamentos. Realizou-se análise estatística descritiva e inferencial dos dados. Resultados: $86,9 \%$ dos idosos eram aderentes à terapêutica medicamentosa. Houve associação entre a variável adesão e as variáveis comportamentais "acreditar que os medicamentos são importantes para manutenção da saúde" e "ter vontade de não tomar os medicamentos". Os idosos que não acreditavam na importância do uso dos medicamentos, não sabiam o nome destes, usavam-nos somente na quando apresentavam sintomas, esqueciam-se de utilizá-los ou não seguiam a prescrição conforme a indicação médica, tinham menor mediana de adesão do que os que não apresentavam esses comportamentos. Conclusão: fatores comportamentais apresentam associação com a adesão à terapêutica medicamentosa em idosos em atendimento ambulatorial. É importante identificar os fatores comportamentais que podem interferir no uso de medicamentos por idosos porque eles podem ser modificados, por meio de ações com vistas a promover a adesão medicamentosa.

Descritores: Adesão à medicação; Idoso; Saúde do idoso; Assistência ambulatorial; Enfermagem.

\begin{abstract}
Objective: to evaluate the relationship between behavioral factors and adherence to drug therapy in elderly patients in outpatient care. Methods: an exploratory, descriptive, cross - sectional, and quantitative study was carried out with a sample of 107 elderly patients in outpatient care at a university hospital. The data were collected by means of interviews to evaluate: instrument of characterization of the elderly, mini-mental state examination, and measure of adherence to the treatment. A descriptive and inferential statistical analysis was performed. Results: a total of $86.9 \%$ of the elderly were adherent to the drug therapy. There was association between the adherence variable and the behavioral variables "believing that medicines are important for maintaining health" and "being willing to not take the medicines". The elders who did not believe in the importance of using medications did not know the medicine names; they used drugs only when there were the presence symptoms; they forgot to use the medicines or did not follow the prescription according to the medical indication; they had a lower average adherence rate than those who did not present these behaviors. Conclusion: behavioral factors are associated with adherence to drug therapy in the elderly in ambulatory care. It is important to identify the behavioral factors that might interfere in the use of drugs by the elderly since it can be modified through actions that would promote more adherence.
\end{abstract}

Descriptors: Medication adherence; Aged; Health of the elderly; Ambulatory Care; Nursing.

\section{RESUMEN}

Objetivo: evaluar la relación entre los factores conductuales y la adhesión al tratamiento farmacológico en los ancianos en la atención ambulatoria. Método: estudio exploratorio, descriptivo, transversal, cuantitativo, con muestra de 107 ancianos en atención ambulatoria en un hospital universitario. Los datos fueron recolectados por medio de una entrevista, cuando se aplicaron: instrumento de caracterización de los ancianos, mini examen del estado mental y medida de adhesión a los tratamientos. Se realizaron análisis estadísticos descriptivos e inferenciales de los datos. Resultados: $86,9 \%$ de los ancianos fueron adherentes a la farmacoterapia. Ha sido identificado una asociación entre la adhesión variable y las variables conductuales "creyendo que los fármacos son importantes para el mantenimiento de la salud" y "queriendo no tomar los fármacos". Los ancianos que no creían en la importancia del uso de fármacos, tampoco sabían sus nombres, sólo se los usaban en el momento en que mostraron síntomas, se olvidaron de usarlos o no siguieron la prescripción de acuerdo con la indicación médica, tuvieron menor media que los que no presentaron estos comportamientos. Conclusión: los factores del comportamiento presentan la asociación con la adhesión a la terapia con fármacos en los ancianos en atención ambulatoria. Es importante identificar los factores conductuales que pueden intervenir con el uso de fármacos por los ancianos porque pueden ser modificados a través de acciones con el fin de promover la adhesión.

Descriptores: Cumplimiento de la medicación; Anciano; Salud del anciano; Atención Ambulatoria; Enfermería.

${ }^{1}$ Enfermeira. Doutora, Escola de Enfermagem, Universidade Federal do Rio Grande. ${ }^{2}$ Enfermeira. Doutora em Enfermagem. Docente aposentada da Escola de Enfermagem, Universidade Federal do Rio Grande. ${ }^{3}$ Enfermeiro. Doutor em Enfermagem. Docente do Centro Universitário Franciscano. ${ }^{4}$ Enfermeira. Doutora, Escola de Enfermagem, Universidade Federal do Rio Grande. ${ }^{5}$ Enfermeira. Especialista em Saúde da Família. Doutoranda do Programa de Pós-Graduação em Enfermagem, Universidade Federal do Rio Grande. ${ }^{6}$ Graduanda em Enfermagem, Universidade Federal do Rio Grande.

\section{Como citar este artigo:}

Abreu DPG, Santos SSC, Ilha S, et al. Fatores Comportamentais Associados à Adesão Medicamentosa Em Idosos Em Atendimento Ambulatorial. Revista de Enfermagem do Centro Oeste Mineiro. 2019;9: e3025. [Access ; Available 


\section{INTRODUÇÃO}

As condições crônicas não transmissíveis constituem um problema de magnitude marcante, afetando os seguimentos de maior idade. Elas representam cerca de $80 \%$ da carga de doenças, em todo o mundo, sendo responsáveis por duas a cada três mortes, e respondem por mais de $70 \%$ das causas de mortes, no Brasil ${ }^{1}$.

O tratamento básico para a maioria das condições crônicas consiste na educação em saúde, modificações dos hábitos de vida e, se necessário, uso de medicamentos. Nesse sentido, a adesão medicamentosa pode ser definida como a medida em que o indivíduo toma medicamentos prescritos, de acordo com a dosagem e o intervalo recomendados por um provedor de saúde ${ }^{2}$.

Em relação especificamente à adesão ao tratamento medicamentoso, estudos indicam que, no Brasil, ela é baixa na população de um modo geral ${ }^{3-4}$. Estudo realizado para descrever e analisar o perfil das pessoas com DM no Brasil, encontrou um baixo percentual de adesão (2\%) aos hipoglicemiantes orais ${ }^{3}$.

Já em estudo realizado em um município no noroeste do Rio Grande do Sul, no que se refere à classificação da adesão ao tratamento da HAS, 20\% eram aderentes ao tratamento., e 67 $(46,2 \%)$ são prováveis aderentes ${ }^{4}$. Em ambos os estudos, a avaliação da adesão foi realizada a partir das perguntas do Brief Medication Questionnaire $^{3-4}$.

As pessoas idosas geralmente apresentam mais de uma condição crônica e necessitam utilizar um número maior de medicamentos para tratá-las. O uso de múltiplos medicamentos pode tornar a adesão medicamentosa mais difícil para pessoas idosas, sendo os índices de baixa adesão à medicação piores na população idosa do que na população geral, podendo chegar a mais de $80 \%^{5-6}$.

A adesão é um fenômeno multidimensional, determinado por vários fatores, entre eles: socioeconômicos, do paciente, da doença, dos profissionais e do sistema de saúde ${ }^{2}$. Dentro dos fatores ligados ao paciente, estão os comportamentais, como: as crenças a respeito dos medicamentos; o conhecimento e entendimento a respeito da própria condição de saúde e do tratamento medicamentoso e não medicamentoso ${ }^{7-8}$.

Algumas consequências da não adesão aos medicamentos prescritos são: agravamento e prolongamento do curso das enfermidades, aumento da morbidade e mortalidade; aumento do número de hospitalizações; aumento dos gastos com saúde; sentimentos de frustração dos profissionais de saúde; sentimento de fracasso dos pacientes, dos familiares e da sociedade em geral $^{9-10}$.

Estudos internacionais têm reportado a relação entre fatores comportamentais e adesão aos medicamentos em idosos ${ }^{7-8}$. No Brasil, poucas pesquisas abordam os fatores comportamentais ${ }^{11-12}$. Desse modo, conhecer os fatores comportamentais associados à adesão aos medicamentos prescritos pode facilitar o desenvolvimento de ações que promovam a adesão em idosos, o que justifica o desenvolvimento deste estudo.

Face ao exposto e, tendo em vista a lacuna do conhecimento nesta temática, objetivou-se avaliar a relação entre fatores comportamentais e adesão à terapêutica medicamentosa em idosos em atendimento ambulatorial.

\section{MÉTODO}

Estudo transversal, oriundo do projeto "Adesão à terapêutica medicamentosa e fatores relacionados em idosos em atendimento ambulatorial", realizado nos ambulatórios de Angiologia, Cardiologia, Pneumologia, Endocrinologia, Gastroenterologia e Urologia de um hospital universitário no Rio Grande, Rio Grande do Sul, Brasil. O estudo foi elaborado segundo recomendações STROBE ${ }^{13}$. O projeto de pesquisa foi desenvolvido, após ser aprovado por um Comitê de Ética em Pesquisa, com parecer favorável número 164/2013.

A amostra do estudo foi constituída por pessoas idosas, consideradas segundo o Estatuto do Idoso ${ }^{14}$ como aquelas com 60 anos e mais, em atendimento ambulatorial, para as quais foram aplicados os seguintes critérios de inclusão: estar em atendimento ambulatorial no hospital universitário; fazer uso de no mínimo um medicamento por pelo menos 15 dias anteriores ao dia da entrevista.

Foram critérios de exclusão do estudo: estar em tratamento com quimioterápicos ou com radioterapia, devido às características específicas desses tratamentos que podem interferir na adesão medicamentosa ${ }^{(15)}$; apresentar discurso desconexo com perdas importantes de memória que impedissem a resposta às questões dos instrumentos de pesquisa. 
A amostra foi estimada de acordo com a fórmula para população infinita em estudos transversais: $\mathrm{n}=\left((\mathrm{Z} \alpha / 2)^{2}\right.$.P.Q $) / \mathrm{E}^{2}$. Onde: $Z \alpha-$ nível de significância do estudo de $95 \%(1,96)$; P prevalência do evento de $50 \%$ que maximiza o tamanho da amostra quando não se sabe a prevalência; Q - complementar da prevalência (1P); $\mathrm{E}$ - erro amostral de $10 \%$. Obteve-se $\mathrm{n}=96$, sendo adicionados $20 \%$ de participantes para controle de fatores de confusão e perdas.

Os dados foram coletados no mês de novembro de 2013, por meio de entrevista, na qual foram utilizados três instrumentos. $O$ primeiro foi elaborado pela pesquisadora e tinha como objetivo caracterizar o idoso quanto ao sexo, faixa etária, estado marital, escolaridade, renda, condições crônicas, número de medicamentos utilizados e também quanto às variáveis comportamentais (dúvida_medicamentos, crença_medicamentos, nome_medicamentos, função_medicamentos, interrupção_tratamento, álcool, medicar_sintomas, tratamento_alternativo, esquecimento, vontade_medicar, seguimento_tratamento). $O$ instrumento foi submetido à validade de face e de conteúdo junto a dois docentes que desenvolviam estudos na área de Gerontologia e eram membros do Grupo de Estudos e Pesquisa em Gerontogeriatria, Enfermagem/Saúde e Educação (GEP-GERON).

Após realizar as alterações sugeridas pelos docentes, o instrumento foi aplicado sob a forma de teste piloto em dez idosos em condições semelhantes às avaliadas no estudo. Não foram percebidas inadequações no teste e considerouse $o$ instrumento adequado. Esses instrumentos não foram incluídos na amostra da pesquisa.

O segundo instrumento utilizado na coleta de dados, denominado Miniexame do Estado Mental (MEEM), serviu para avaliar a cognição do idoso. Foi utilizada uma versão do MEEM validada no Brasil, na qual o ponto de corte utilizado para indicar déficit cognitivo foi de 18 pontos para analfabetos e 23 pontos para aqueles com mais de um ano de escolaridade ${ }^{16}$.

O terceiro instrumento, denominado Medida de Adesão aos Tratamentos (MAT), foi utilizado para verificar a adesão do idoso à terapêutica medicamentosa, tendo sido construído e validado em Portugal ${ }^{17}$. Esse é composto por sete itens, cujas respostas são sob a forma de escala Likert, e as pontuações variam de sempre $=1$ até nunca $=6$. As respostas de cada um dos itens são somadas e esse valor é dividido pelo número total de itens. O valor obtido é convertido em uma escala dicotômica na qual os valores entre 1 e 4 são considerados como não adesão ao tratamento e os entre 5 e 6 como adesão. $\mathrm{O}$ alpha de Cronbach da escala foi de $0,66^{17}$.

As entrevistas estruturadas foram realizadas por integrantes do GEP-GERON que receberam capacitação. Cada idoso foi abordado na sala de espera dos ambulatórios por uma entrevistadora antes ou após a consulta médica. Antes da realização da entrevista, solicitou-se o consentimento do idoso e este foi esclarecido a respeito da pesquisa. Aquele que aceitou participar assinou ou colocou a digital em duas vias do Termo de Consentimento Livre e Esclarecido, sendo que uma via foi entregue ao participante e outra ficou com a pesquisadora.

Também foi informado ao participante que o risco para ele foi considerado mínimo uma vez que não houve intervenção e que a pesquisa pode gerar benefícios aos mesmos, através dos conhecimentos que serão produzidos a partir dela. O estudo seguiu todos os preceitos da resolução 466/2012 que orienta a realização de pesquisas com seres humanos ${ }^{18}$.

Para a organização dos dados, foi elaborada uma planilha no programa Microsoft ${ }^{\circledR}$ Excel 2007. A análise dos dados teve o auxílio do programa Statistical Package for the Social Sciences ${ }^{\circledR}$ (SPSS) versão 20.0.

Foram utilizados procedimentos da estatística descritiva com a apresentação das frequências simples e percentuais das variáveis categóricas (sexo, faixa etária, estado marital, escolaridade e renda) e medidas de tendência central e de dispersão para as variáveis numéricas (MAT e número medicamentos). Na análise inferencial, foram realizados testes para verificar a associação entre as variáveis.

O teste de Mann-Whitney foi utilizado para verificar a associação entre as variáveis comportamentais presentes no instrumento de caracterização dos participantes (dúvida_medicamentos, crença_medicamentos, nome_medicamentos, função_medicamentos, interrupção_tratamento, álcool, medicar_sintomas, tratamento_alternativo, esquecimento, vontade_medicar, seguimento_tratamento) e a mediana de adesão que foi obtida por meio do instrumento MAT.

Para verificar a associação entre a variável adesão obtida por meio do instrumento MAT (aderente ou não aderente) e as variáveis 
relacionadas aos fatores comportamentais presentes no instrumento de caracterização dos participantes (dúvida_medicamentos, crença_medicamentos, nome_medicamentos, função_medicamentos, interrupção_tratamento, álcool, medicar_sintomas, tratamento_alternativo, esquecimento, vontade_medicar, seguimento_tratamento) e o MEEM, utilizou-se para frequências esperadas maiores de 5, o teste de Qui-Quadrado e, para frequências esperadas menores de 5 , o teste Exato de Fisher. Para as análises inferenciais, foi considerado o nível de significância de $p<0,05$.

\section{RESULTADOS E DISCUSSÃO}

Dos 116 idosos investigados, nove devolveram os instrumentos respondidos de forma incompleta e a amostra final foi composta por 107 participantes. Entre os participantes, houve predomínio de idosos: do sexo feminino; na faixa etária entre 60-69 anos; que tinham companheiro; possuíam entre 1 e 4 anos de estudo; com renda entre 1 e 3 salários mínimos, conforme Tabela 1.

Tabela 1 - Distribuição dos idosos em atendimento ambulatorial de acordo com as variáveis demográficas e socioeconômicas. Rio Grande, RS, Brasil, 2013 ( $n=107)$.

\begin{tabular}{lll}
\hline Variáveis demográficas e socioeconômicas & $\mathbf{N}$ & $\%$ \\
\hline Sexo & 74 & 69,2 \\
$\quad$ Feminino & 33 & 30,8 \\
Masculino & & 66,4 \\
Faixa etária & 71 & 26,1 \\
$60-69$ anos & 28 & 7,5 \\
$70-79$ & 8 & 59,8 \\
80 e mais & & 40,2 \\
Estado marital & 64 & 9,3 \\
Com companheiro & 43 & 42,1 \\
Sem companheiro & & 32,7 \\
Escolaridade & 10 & 15,9 \\
Analfabetos & 45 & 27,1 \\
1 a 4 anos de estudo & 35 & 58,9 \\
5 a 8 anos de estudo & 17 & 7,5 \\
mais de 8 anos de estudo & & 6,5 \\
Renda† & 29 & 63 \\
Até 1 salário mínimo & 8 & 7 \\
Mais de 1 até 3 salários mínimos & & \\
Mais de 3 salários mínimos & & \\
Não informaram a renda & & \\
\hline \hline
\end{tabular}

†Salário mínimo em 11/2013 no Brasil = R\$ 672,00.

Fonte: Dados da pesquisa.

O perfil demográfico e socioeconômico dos idosos do presente estudo foi semelhante aos encontrados em outras pesquisas acerca da adesão, realizadas com idosos no Brasi ${ }^{6,11,19}$. Ele reflete a feminização do envelhecimento, a presença da maioria dos idosos nas faixas etárias iniciais da velhice e a baixa escolaridade destes.

O resultado no MEEM mostrou que 93 $(86,9 \%)$ idosos apresentaram resultado considerado normal na avaliação e 14 (13,1\%) apresentaram resultado sugestivo de déficit cognitivo. O MEEM foi aplicado com o intuito de verificar se o estado cognitivo estava interferindo na adesão, o que poderia representar um viés para o estudo. Contudo, não houve associação entre adesão e o resultado do MEEM no Teste Exato de Fischer $(p=1,000)$.
As condições crônicas mais prevalentes nas pessoas idosas entrevistadas foram a HAS presente em 85 (79,4\%), o DM em 61 (57,0\%), as cardiopatias em $47(43,9 \%)$, a artrite em 37 $(34,6 \%)$. Outras condições crônicas foram referidas pelas pessoas idosas, mas não foram descritas por serem prevalentes em menos de $20 \%$ dos entrevistados. Essas condições também foram as mais prevalentes em outro estudo realizado com população idosa ${ }^{20}$.

Os idosos utilizavam em média 4,8 medicamentos, semelhantes ao encontrado em estudo realizado com idosos hospitalizados em Cuiabá-MT, em que mais de $40 \%$ da amostra utilizava entre 4 a mais de 6 medicamentos ${ }^{19}$. Embora a média tenha sido semelhante ao encontrado em outro estudo com população idosa, cabe ressaltar que o uso de vários 
medicamentos (cinco ou mais) configura polifarmácia e pode expor o idoso a um maior risco de interações medicamentosas, reações adversas e não adesão à medicação ${ }^{4}$.

Dos 107 participantes do estudo, 93 $(86,9 \%)$ obtiveram valor igual ou superior a 5 na escala MAT, sendo considerados aderentes à terapêutica medicamentosa prescrita. A mediana de adesão na MAT foi de 5,42 (P25 = 5,14 e P75 = $5,71)$. Estudos realizados com pessoas idosas no Brasil demonstram resultados diferentes. No mesmo estudo de Cuiabá-MT, 82,7\% apresentaram baixo grau de adesão ${ }^{19}$. Em outro estudo realizado com idosos com hipertensão em atenção primária em Ribeirão Preto - SP, mostrou que $81,2 \%$ eram menos aderentes ${ }^{5}$.

Na Tabela 2, descreve-se a prevalência de idosos aderentes e não aderentes em relação aos fatores comportamentais e o valor de $p$ obtido no teste de associação. A variável crença medicamentos apresentou associação estatisticamente significante com a variável adesão $(p=0,001)$. A variável vontade medicar também apresentou associação estatisticamente significante com a variável adesão $(p=0,004)$. As demais variáveis comportamentais não apresentaram associação significativa com a variável adesão medicamentosa.

Tabela 2 - Prevalência de adesão à terapêutica medicamentosa prescrita em idosos em atendimento ambulatorial de acordo com os fatores comportamentais. Rio Grande, RS, Brasil, 2013 ( $n=107)$.

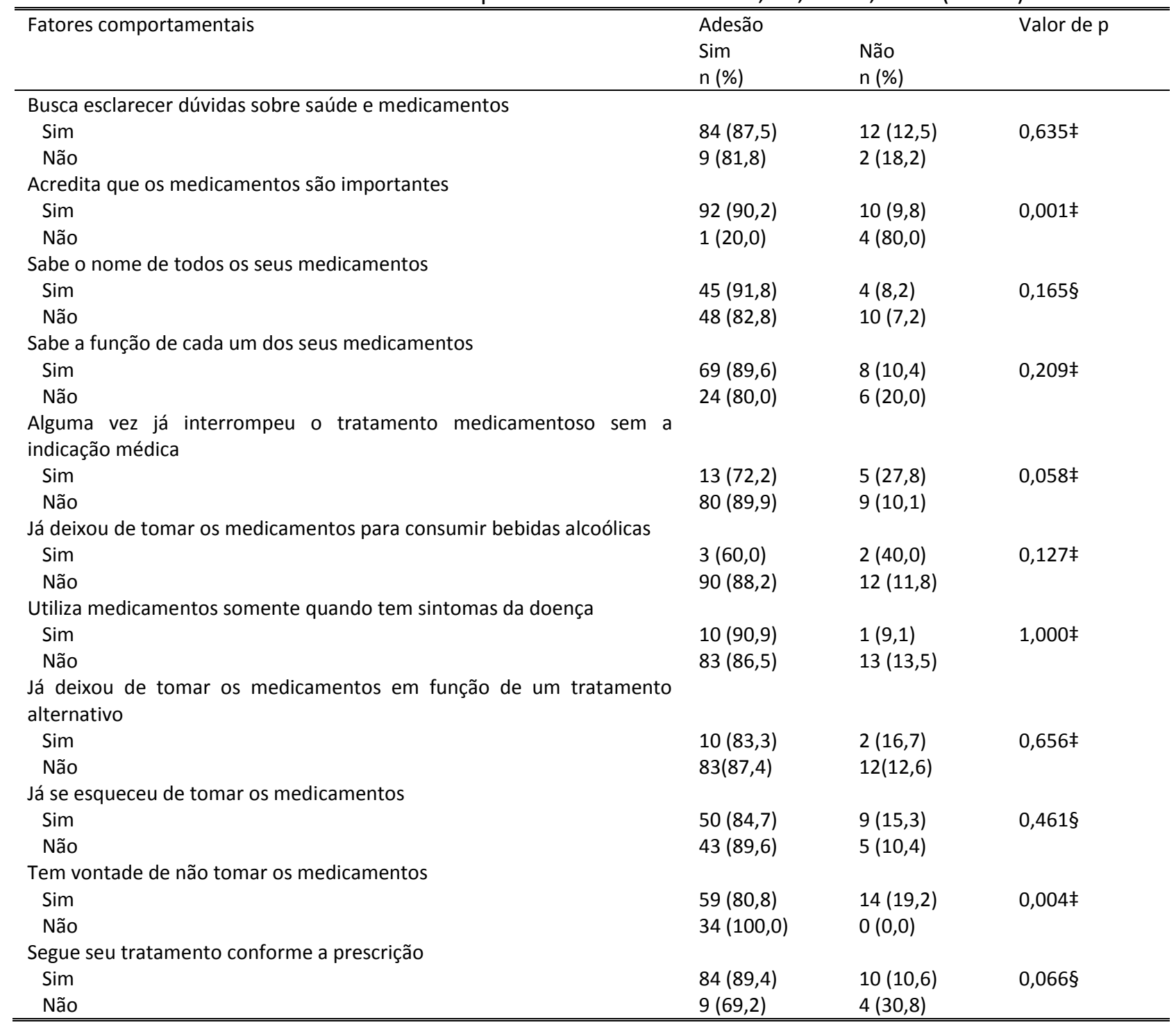

¥Teste Exato de Fischer §Teste Qui-Quadrado.

Fonte: Dados da pesquisa.

Verificou-se, por meio do teste de MannWhitney, que os idosos que não acreditavam na importância do uso dos medicamentos, não sabiam o nome destes, utilizavam-nos somente na presença de sintomas, esqueciam-se de utilizálos ou não seguiam a prescrição conforme a 
indicação médica, tinham menor mediana na MAT do que os que não apresentavam esses comportamentos, sendo tal diferença estatisticamente significante, conforme Tabela 3.

Tabela 3 - Valores da mediana da Medida de Adesão aos Tratamentos de idosos em atendimento ambulatorial de acordo com os fatores comportamentais. Rio Grande, RS, Brasil, 2013 ( $n=107)$.

\begin{tabular}{|c|c|c|}
\hline Fatores comportamentais & $\begin{array}{l}\text { Mediana } \\
\text { MAT++ }\end{array}$ & $\begin{array}{l}\text { Valor } \\
\text { pł‡ }\end{array}$ \\
\hline \multicolumn{3}{|c|}{ Busca esclarecer dúvidas sobre saúde e medicamentos } \\
\hline Sim & 5,42 & 0,959 \\
\hline Não & 5,42 & \\
\hline \multicolumn{3}{|c|}{ Acredita que os medicamentos são importantes } \\
\hline Sim & 5,42 & 0,003 \\
\hline Não & 4,28 & \\
\hline \multicolumn{3}{|c|}{ Sabe o nome de todos os seus medicamentos } \\
\hline Sim & 5,57 & 0,006 \\
\hline Não & 5,35 & \\
\hline \multicolumn{3}{|c|}{ Sabe a função de cada um dos seus medicamentos } \\
\hline Sim & 5,42 & 0,062 \\
\hline Não & 5,28 & \\
\hline \multicolumn{3}{|c|}{ Alguma vez já interrompeu o tratamento medicamentoso sem a indicação médica } \\
\hline Sim & 5,42 & 0,275 \\
\hline Não & 5,42 & \\
\hline \multicolumn{3}{|c|}{ Já deixou de tomar os medicamentos para consumir bebidas alcoólicas } \\
\hline Sim & 5,42 & 0,489 \\
\hline Não & 5,42 & \\
\hline \multicolumn{3}{|c|}{ Utiliza medicamentos somente quando tem sintomas da doença } \\
\hline Sim & 5,28 & 0,047 \\
\hline Não & 5,42 & \\
\hline \multicolumn{3}{|c|}{ Já deixou de tomar os medicamentos em função de um tratamento alternativo } \\
\hline Sim & 5,28 & 0,101 \\
\hline Não & 5,42 & \\
\hline \multicolumn{3}{|c|}{ Já se esqueceu de tomar os medicamentos } \\
\hline Sim & 5,28 & 0,000 \\
\hline Não & 5,71 & \\
\hline \multicolumn{3}{|c|}{ Tem vontade de não tomar os medicamentos } \\
\hline Sim & 5,42 & 0,119 \\
\hline Não & 5,42 & \\
\hline \multicolumn{3}{|c|}{ Segue seu tratamento conforme a prescrição } \\
\hline Sim & 5,42 & 0,019 \\
\hline Não & 5,14 & \\
\hline
\end{tabular}

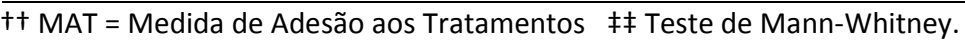

Fonte: Dados da pesquisa.

Em relação aos fatores comportamentais, a maioria dos idosos aderentes buscava esclarecer as suas dúvidas sobre os medicamentos. Pacientes com mais conhecimento sobre os medicamentos prescritos, bem como sobre os comportamentos requeridos para o seguimento do tratamento parecem ser mais prováveis de aderir à medicação do que aqueles com menos informação ${ }^{21}$.

No presente estudo, a crença de que os medicamentos são importantes para a manutenção da saúde apresentou associação com a adesão. Corroborando esse achado, pesquisa realizada em ambulatório geral de adultos de um hospital universitário de grande porte no interior do estado de São Paulo também encontrou essa relação ${ }^{12}$. As crenças positivas a respeito dos medicamentos podem estar relacionadas à percepção dos benefícios destes, à medida que os idosos os utilizam e têm melhora em seu quadro de saúde ${ }^{6}$.

Os idosos aderentes apresentaram maior conhecimento sobre o nome e função dos medicamentos que utilizavam que os não aderentes. Saber sobre o nome, função, dose e frequência de administração dos medicamentos é de suma importância para que o idoso evite cometer erros na utilização que podem interferir na adesão. Além disso, favorece a autonomia do idoso, bem como possibilita maior segurança para o tratamento em saúde. A falta de conhecimento sobre a condição crônica, o tratamento e os 
medicamentos mostraram-se relacionados a não adesão em outras investigações ${ }^{12,19}$.

Entre os idosos entrevistados, os que já haviam interrompido alguma vez o tratamento sem a indicação do prescritor apresentavam maior prevalência de não adesão. É importante entender o que levou o idoso a interromper o tratamento, pois esse comportamento compromete a adesão e pode contribuir para que ocorram complicações no seu estado de saúde. Em estudo realizado com 100 idosos em acompanhamento ambulatorial, em um hospital universitário, no interior de São Paulo, encontrou que, interromper o uso dos medicamentos por se sentir pior, explicou 12,8 e $13,5 \%$ da variabilidade da proporção de adesão aos antihipertensivos e aos antidiabéticos orais/insulina, respectivamente ${ }^{11}$.

A ausência de sintomas da condição crônica também pode levar o idoso a interromper o tratamento por achar que não necessita dos medicamentos, pois está se sentindo bem. A ausência de sintomas da condição crônica pode significar para alguns a inexistência de problemas de saúde e, em consequência disso, as pessoas idosas deixam de utilizar os medicamentos prescritos, pois acreditam que eles são desnecessários ${ }^{22}$.

Entre os idosos investigados neste estudo, havia os que tomavam os medicamentos somente quando apresentavam sintomas da condição crônica de saúde. Estes apresentaram menor nível de adesão do que aqueles que seguiam o tratamento de forma contínua. A não conscientização por parte do idoso da importância do seguimento da terapia pode refletir em piora na condição crônica ${ }^{19}$. Nesse sentido, faz-se necessária a realização, por parte dos profissionais de saúde e da enfermagem, de atividades de educação em saúde para os idosos e familiares em relação às condições crônicas, ao tratamento e aos medicamentos.

Dentre as pessoas idosas entrevistadas nenhuma fazia uso de cigarro e cinco $(4,6 \%)$ faziam uso de bebidas alcoólicas. No presente estudo, o consumo de álcool não apresentou associação com a adesão.

No entanto, estudos nacionais e internacionais têm destacado que o consumo de bebidas alcoólicas interferem na adesão ${ }^{20,23}$. 0 consumo de bebidas alcoólicas é um fato que deve ser atentado pelos profissionais de saúde, pois, além de interferirem na adesão à medicação, trazem prejuízos como o aumento de pressão, colesterol, triglicerídeos e de peso, podendo agravar o estado de saúde das pessoas idosas $^{20}$.

No presente estudo, os idosos que já haviam deixado de tomar os medicamentos em função de um tratamento alternativo apresentaram menor nível de adesão do que os que não apresentaram esse comportamento. Contudo, não houve nenhuma associação significativa entre o uso de tratamento alternativo e a variável adesão. O mesmo achado foi encontrado em estudo realizado com hipertensos e diabéticos em uma unidade de saúde da família, no município de Itabuna - $B A^{23}$.

Entre os entrevistados, os que já haviam se esquecido, alguma vez, de tomar os medicamentos apresentaram menor mediana da MAT do que os que não esqueceram. Pesquisas também relatam o esquecimento como um dos principais fatores relacionados a não adesão à terapêutica medicamentosa ${ }^{4,12}$. O esquecimento pode ocorrer em decorrência, entre outros fatores, da polifarmácia, dos transtornos mentais e dos prejuízos cognitivos, que podem dificultar o reconhecimento e memorização dos horários de administração dos medicamentos ${ }^{4}$.

Entre os idosos não aderentes todos referiram não ter vontade de tomar os medicamentos e a variável apresentou associação significativa com a variável adesão. O enfermeiro deve procurar saber os motivos que levam o idoso a não querer tomar os medicamentos para, a partir de então, planejar ações que possam minimizar/evitar a não adesão.

No presente estudo, os idosos que relataram seguir a prescrição conforme a orientação médica (dose, frequência e horário), apresentaram a mediana da MAT maior do que os que relataram não seguir a prescrição conforme a orientação médica. A carência de informações sobre as condições crônicas de saúde e a baixa percepção dos benefícios advindos com o uso dos medicamentos podem gerar dúvidas quanto à necessidade e eficácia do tratamento medicamentoso prescrito e podem colaborar para a não adesão medicamentosa ${ }^{6}$.

Como limitação deste estudo, tem-se o tamanho reduzido da amostra selecionada de forma consecutiva por conveniência. Como ponto forte 0 estudo identificou que fatores comportamentais estão associados à adesão à terapêutica medicamentosa e que devem ser considerados pelos profissionais de 
saúde/enfermeiro no planejamento de suas ações com o paciente idoso.

\section{CONCLUSÃO}

Houve associação entre a adesão e a crença de que os medicamentos são importantes para manutenção da saúde e ter vontade de não tomar os medicamentos. Idosos que não acreditavam na importância do uso dos medicamentos, não sabiam o nome dos medicamentos que utilizavam, usavam-nos somente quando apresentavam sintomas, se esqueciam de utilizar os medicamentos ou não seguiam a prescrição conforme a indicação médica, tiveram menor mediana de adesão na MAT do que os que não apresentaram essas condições.

Como contribuição do estudo para a prática da enfermagem, enfatiza-se a importância de identificar os fatores comportamentais que podem interferir no uso de medicamentos por idosos, tendo em vista que eles podem ser modificados através de ações com vistas a favorecer a adesão. Nesse sentido, os resultados do estudo podem servir de subsídio para o planejamento da assistência de enfermagem a pessoas idosas em atendimento ambulatorial e que utilizam medicamentos.

\section{REFERÊNCIAS}

1 - Instituto Brasileiro de Geografia e Estatística (IBGE). Projeção da população do Brasil por sexo e idade para o período 2000/2060. Projeção da população das Unidades da Federaçã opor sexo e idade para o período 2000/2030.

Brasília: IBGE; 2013.

2 - Brasil. Ministério da Saúde. Síntese de evidências para políticas de saúde: Adesão ao tratamento medicamentoso por pacientes portadores de doenças crônicas. Brasília: Ministério da Saúde; 2016.

3 - Meiners MMMA, Tavares MUL, Guimarães LSP, Bertoldi AD, Dal Pizzol TS, Luiza VL et al. Acesso e adesão a medicamentos entre pessoas com diabetes no Brasil: Evidências da PNAUM. Rev Bras Epidemiol. 2017;20(3):445-59. DOI: 10.1590/1980-5497201700030008

4 - Gewehr DM, Bandeira VAC, Gelatti GT, Colet $\mathrm{CF}$, Oliveira KR. Adesão ao tratamento farmacológico da hipertensão arterial na Atenção Primária à Saúde. Saúde Debate 2018;42(116):179-90. DOI: $10.1590 / 0103-$ $\underline{1104201811614}$
5 - Vieira LB, Cassiani SHB. Avaliação da adesão medicamentosa de pacientes idosos hipertensos em uso de polifarmácia. Rev Bras Cardiol. 2014 [citado em 15 dez 2017]; 27(3):195-202. Disponível em: http://www.rbconline.org.br/wpcontent/uploads/Art 181 Liliana Vieira Artigo Original.pdf

6 - Borba AKDOT, Marques APDO, Ramos VP, Leal MCC, Arruda IKGD, Ramos RSPDS. Fatores associados à adesão terapêutica em idosos diabéticos assistidos na atenção primária de saúde. Ciênc Saúde Coletiva 2018;23(3):953-61. DOI: $10.1590 / 1413-81232018233.03722016$

7 - Rajpura JR, Nayak R. Role of illness perceptions and medication beliefs on medication compliance of elderly hypertensive cohorts. J Pharm Pract. 2014;27(1):19-24.

DOI: 10.1177/0897190013493806

8 - Bae SG, Kam S, Park KS, Kim KY, Hong NS, Kim KS et al. Factors related to intentional and unintentional medication nonadherence in elderly patients with hypertension in rural community. Patient Prefer Adherence 2016; 10:1979-89. DOI: 10.2147/PPA.S114529

9 - Kim S, Shin DW, Yun JM, Hwang J, Park SK, Ko $\mathrm{YJ}$ et al. Medication adherence and the risk of cardiovascular mortality and hospitalization among patients with newly prescribed antihypertensive medications. Hypertension 2016;67(3):506-12.

DOI:

10.1161/HYPERTENSIONAHA.115.06731

10 - Yang Z, Howard DH, Will J, Loustalot F, Ritchey M, Roy K. Association of antihypertensive medication adherence with healthcare use and medicaid expenditures for acute cardiovascular events. Med Care 2016;54(5):504-11. DOI: 10.1097/MLR.0000000000000515

11 - Jannuzzi FF, Cintra FA, Rodrigues RCM, SãoJoão TM, Gallani MCBJ. Adesão medicamentosa e qualidade de vida em idosos com retinopatia diabética. Rev Latino-Am Enfermagem 2014;22(6):902-10. DOI: 10.1590/01041169.3477 .2494

12 - Jannuzzi FF, Rodrigues RCM, Cornélio ME, São-João TM, Gallani MCBJ. Beliefs related to adherence to oral antidiabetic treatmentaccording to the Theory of Planned Behavior. Rev Latino-Am Enfermagem 2014;22(4):529-37. DOI: 10.1590/01041169.3578 .2448

13 - Malta M, Cardoso LO, Bastos Fl, Magnani MMF, Silva CMFP. Iniciativa STROBE: Subsídios para a comunicação de estudos observacionais. 
Rev Saúde Pública 2010;44(3):559-65. DOI: $\underline{10.1590 / \text { S0034-89102010000300021 }}$

14 - Brasil. Decreto no 1.948, de 3 de julho de 1996. Regulamenta a Lei 8.842 , de 4 de janeiro de 1994, a qual dispõe sobre a Política Nacional do Idoso, e dá outras providências. Diário Oficial da União 1996;1:12277.

15 - Gautério-Abreu DP, Santos SSC, Silva BT, Ilha $\mathrm{S}$, Gomes GC. Pessoas idosas em atendimento ambulatorial: Motivos que levam a adesão/não adesão aos medicamentos. Texto Contexto Enferm. 2015;24(4):1094-103. DOI: 10.1590/0104-0707201500002230014

16 - Lourenço RA, Veras RP, Ribeiro PCC. Confiabilidade teste-reteste do Mini-Exame do Estado Mental em uma população idosa assistida em uma unidade ambulatorial de saúde. Rev Bras Geriatr Gerontol. 2008 [citado em 19 dez 2017]; 11(1):7-16. Disponível em: http://revista.unati.ueri.br/scielo.php?script=sci arttext\&pid=S180998232008000100002\&lng=pt\& nrm=iso\&tlng=pt

17 - Delgado $A B$, Lima ML. Contributo para validação concorrente de uma medida de adesão aos tratamentos. Psic, Saúde \& Doenças 2001 [citado em 19 dez 2017]; 2(2):81-100. Disponível em:

http://www.scielo.mec.pt/pdf/psd/v2n2/v2n2a06 .pdf

18 - Conselho Nacional de Saúde (Brasil). Resolução $n^{\circ}$ 466, de 12 de dezembro de 2012. Diário Oficial da União 2012.

19 - Britto AS, Abreu HCA, Rubira EA, Marcon SR, Oliveira JRT. Grau de adesão de idosos internados em um hospital ao regime terapêutico para hipertensão e diabetes. RAS 2015;13(44):27-32. DOI: $10.13037 /$ ras.vol13n44.2582

20 - Han E, Sohn HS, Lee JY, Jang S. Health behaviors and medication adherence in elderly patients. Am J Health Promot. 2017;31(4):278-86. DOI: 10.4278/ajhp.150205-QUAN-709

21 - Pereira MV, Alencar JS, Souto RP, Pinto NB, Saraiva SEM. Grau de conhecimento dos pacientes sobre o tratamento: Estratégia para uso racional de medicamentos. J Health NPEPS. 2016 [citado em 19 dez 2017]; 1(1):31-9. Disponível em:

file:///C:/Users/User/AppData/Local/Packages/M icrosoft.MicrosoftEdge 8wekyb3d8bbwe/TempSt ate/Downloads/1557-5417-2-PB\%20(1).pdf 22 - Becho AS, Oliveira JLT, Almeida GBS. Dificuldades de adesão ao tratamento por hipertensos de uma unidade de atenção primária à saúde. Rev APS 2017 [citado em 19 dez 2017];
20(3):349-59. Disponível em: http://webcache.googleusercontent.com/search? $\mathrm{q}=$ cache:9y3VzwvyKZgJ:ojs2.ufjf.emnuvens.com.b r/aps/article/view/15608+\&cd=1\&hl=pt-BR\&ct= clnk\&gl=br

23 - Araújo MCF, Alves MS, Padre PMM, Andrade DM, Silva ACM, Barreto LP et aL. Perfil de não adesão ao Tratamento de Usuários com Diabetes e Hipertensão em uma Unidade de Saúde da Família. Ensaios Cienc, Cienc Biol Agrar Saúde 2016;20(1):43-8. DOI: 10.17921/14156938.2016v20n1p\%25p

Nota: Este artigo é parte da Tese de Doutorado da Escola de Enfermagem da Universidade Federal do Rio Grande, intitulada "Idosos em atendimento ambulatorial: adesão à terapêutica medicamentosa e fatores relacionados". Agencia de fomento: Coordenação de Aperfeiçoamento de Pessoal de Nível Superior (CAPES).

Received in: 25/07/2018

Approved in: 16/03/2019

Endereço de correspondência:

Daiane Porto Gautério Abreu

Rua General Osório, s/n, Área Acadêmica da Saúde - 4응 andar

CEP: 96200400 - Campina Grande/PB - Brasil

E- mail: daianeporto@furg.br 